\title{
artículos
}

\section{Análisis temático documental}

\author{
Blanca Castañón Moreno*
}

\section{RESUMEN}

La definición vaga e imprecisa de lo que se entiende por tema documental es una de las causas principales de las inconsistencias tanto en la operación de análisis temático de documentos, como en la operación de representar temáticamente documentos, es decir, la operación de clasificar, o indizar. Esta imprecisión y vaguedad que se resiente en la mayoría de los sistemas de clasificación e indización tradicionales, también se percibe en los métodos y técnicas que recientemente se han propuesto para que, independientemente de los sistemas de clasificación e indización, o aplicables en cualquier sistema, permitan mejorar y sistematizar la operación de análisis temático. A partir de esos métodos que finalmente han sido extraídos y formalizados en los sistemas tradicionales de clasificación e indización en donde estaban implícitos, este trabajo se propone: a) mostrar esa vinculación entre los sistemas para la representación temática, y los recientes métodos para el análisis, b) con base en esa relación, hacer explícita la concepción temática que permita, comprender mejor, tanto los métodos de análisis que recientemente se están proponiendo, como los sistemas mismos de clasificación e indización.

\begin{abstract}
Defining vaguely and imprecisely just what is meant by the term used in the title of this paper, is one of the main causes of inconsistencies both in the operation of topic analisis of documents and in the operation of representing documents by topics, that is in the operation of classifying or indexing. This vagueness and lack of precision found in the majority of the traditional systems of classifying or indexing also appears in the methods and techniques presented recently to improve and systematize the operation of topic analysis, regardless of the classifying or indexing system involved. Based on these methods, taken from the traditional methods of classifying and indexing, this paper intends to a) show this relationship between the systems based on representation by topic and the more recent methods of analysis, and b) based on this relationship make more explicit the concept of topic which in turn will make it possible to have a better understanding of both the more recant methods of analysis and the systems of classifying and indexing themselves.
\end{abstract}

\section{ANTECEDENTES}

$\mathrm{L}^{\mathrm{s}}$ os cien años que comprenden la segunda mitad del siglo XIX y primera mitad del siglo XX fueron años de esfuerzos notables por crear y desarrollar sistemas eficientes de clasificación e indización, es decir, sistemas para la organización y representación documental teniendo como base las características temáticas de los documentos.

La razón de estos esfuerzos es que los temas, así como los nombres de autores y títulos, han sido las características o elementos de identificación documental más conocidos y los que con mayor fre- cuencia emplean las personas que buscan satisfacer necesidades de conocimiento o información a través de documentos.

Para los bibliotecarios la identificación y representación de autores y títulos no suelen presentar serios problemas en el análisis o examen de cada documento puesto que se trata de elementos que generalmente se dan en la forma relativamente breve y precisa de nombres propios y cuya localización suele estar predeterminada fuera del texto o cuerpo principal de las obras como es la portada, cubiertas, márgenes del texto, o páginas finales de una obra. Estas condiciones han facilitado tanto la sistematización en el análisis, como la determinación y uso de los nombres de autores y títulos como elementos en la descripción, organización y representación documental.

No ocurre lo mismo con el tema de una obra ya que éste se encuentra en todo el texto y su representación y uso con fines de organización y recuperación documental implica la necesidad de sintetizarlo a la brevedad de códigos de clasificación, o palabras que se emplearán como términos de indización, o puntos

* Investigadora del Centro Universitario de Investigaciones Bibliotecológicas 
de acceso en catálogos, bibliografías e índices.

La necesidad de sintetizar la exposición, normalmente extensa, de un tema documental a sus ideas principales y esenciales que posteriormente permitan representarlo breve y objetivamente en códigos o frases, obliga a interpretaciones que no siempre logran ser objetivas y exactas.

La falta de objetividad que lleva a descripciones inexactas o incompletas en las representaciones temáticas dificulta, sino es que impide completamente, la recuperación documental. Aun cuando los problemas de interpretación subjetiva o incorrecta no siempre ocurren e incluso pueden ser más los casos de interpretaciones temáticas correctas, o al menos suficientes y satisfactorias para los usuarios, es innegable que existen condiciones o factores que los bibliotecarios no han controlado y que provocan un desempeño irregular en el análisis par ala determinación temática de documentos.

Entre los factores a los que con mayor frecuencia se atribuyen los errores, omisiones e inconsistencias en el análisis y la posterior representación temática de documentos, suelen estar las limitaciones y obsolescencia de los sistemas de clasificación e indización a cuyas estructuras y símbolos deben traducirse los resultados del análisis temático.

Sin embargo, más que las limitaciones y obsolescencia de los sistemas a los que se suelen atribuir imprecisiones e inconsistencias en la representación temática, muchas veces el problema se genera porque el bibliotecario se preocupa más por ajustarse a un sistema de clasificación o indización que por llevar a cabo un análisis documental que le permita ante todo comprender plenamente el tema que se expone en el texto de una obra.

Derek Langridge advierte que, el análisis temático tiene que ver más con los documentos que con los sistemas, desafortunadamente, en no pocos casos, el examen y análisis de textos para determinar un tema se convierte en la búsqueda de ideas o términos que primero y mejor se acomoden o convengan a las estructuras y símbolos previstos en el sistema que se va a aplicar. No se busca en el análisis la identificación de elementos o características esenciales, sean éstos ideas, conceptos o términos, que caractericen la naturaleza y el alcance total de un tema tal y como se desarrolla en un texto, sino tan sólo aquellas ideas, conceptos, o términos que se supone son elementos que pueden ajustarse y expresarse mejor en las estructuras y símbolos de un sistema determinado de clasificación e indización.

Desde el punto de vista que considera el análisis como una forma de clasificar ya uqe, en cierta manera, el análisis sistemático consiste en "clasificar todos los elementos de un texto, encasillándolos, de modo que como resultado final del texto es caracterizado por el número de los elementos que corresponden a cada una de las casillas" (DUV' 81), es natural y necesario que el análisis temático documental esté rígido por un sistema de clasificación o indización.

El problema de los sistemas de clasificación e indización como rectores de técnicas que pueden emplearse para llevar a cabo el análisis temático no está tanto en las limitaciones o la obsolescencia de sus estructuras y símbolos para ubicar y expresar adecuadas y plenamente temas complejos, muy específicos o nuevos, pues de alguna forma, con mayores o menores recursos, los principales sistemas de clasificación e indización están provistos de los mecanismos para la modificación, la ampliación y la actualización.

El problema fundamental en los sistemas de clasificación e indización es la falta de explicación respecto a lo que esos sistemas entendieron por tema documental, es decir, a qué corresponden y qué representan, por ejemplo, las llamadas "clases principales", subdivisiones, secciones o facetas de las clasificaciones sistemáticas, o qué representan y a qué corresponden los nombres simples o compuestos de los lenguajes de indización.

Los sistemas de clasificación e indización constituyen los primeros intentos por sistematizar el análisis documental para evitar que las bibliotecas tuvieran que reorganizar sus colecciones y catálogos cada vez que cambiaba el bibliotecario, como observaba Melvin Dewey al presentar su clasificación Decimal. Sin

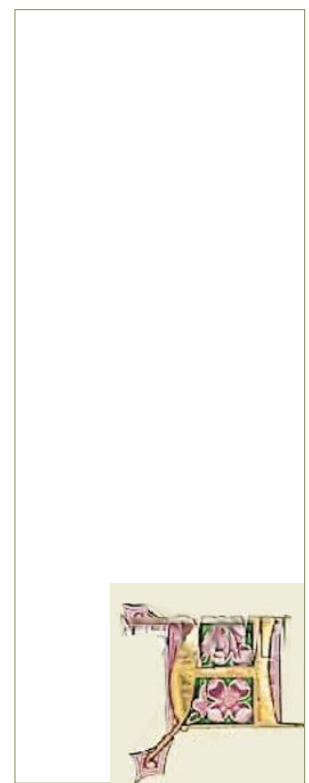

El problema fundamental en los sistemas de clasificación e indización es la falta de explicación respecto a lo que esos sistemas entendieron por tema documental, es decir, a qué corresponden y qué representan 
embargo, los sistemas de organización y representación temática documental como el de Dewey, fallan precisamente en su propósito principal de sistematización, es decir, de un análisis regular y sistemático, al omitir una definición precisa de lo que se está considerando como tema documental dando lugar con ello a que cada quien interprete como quiera o pueda, no solamente lo que busca al examinar un documento, sino las mismas estructuras y símbolos de los sistemas.

Los grandes sistemas de organización y representaci\{on temática documental como la Clasificación Decimal de Dewey, los encabezamientos de materia de charles A. Cutter, la indización sistemática de Julius A. Kaiser y la Library of congress Classification (LCC); sistemas que se dieron en lo que Cutter llamó el final de la “época dorada de la catalogación” cuando la discusión era un placer ("arte perdido" en la bibliotecología), sobreviven agobiados por los errores y malas aplicaciones provocadas por la omisión de definiciones, o explicaciones más claras respecto a sus fundamentaciones teóricas, no obstante lo cual también encierra una gran riqueza acumulada (YOU'90).

Así tenemos que, a pesar de los notables esfuerzos por crear y desarrollar sistemas eficientes de clasificación e indización, así como de una práctica, que en algunos de esos sistemas ya rebasa los cien años de vigencia, persiste un serio problema de sistematización en el análisis y representación temática de documentos que se origina en una concepción vaga e imprecisa de lo que se entiende por tema.

\section{SISTEMATIZACIÓN}

La sistematización en el análisis temático documental se ve actualmente no solamente como una necesidad para mejorar y uniformar criterios en los bibliotecarios, e incluso también en los usuarios cuando éstos interactúan directamente con los sistemas que van a consultar, sino que también se ve como una posibilidad de mejorar, o corregir omisiones o errores evidentes en lo ya hecho en clasificación e indización. Una muestra de esta posibilidad es el índice a la bibliografía nacional británica y para cuya elaboración se ha aplicado el programa
PRECIS (PREserved Context Index) que fundamentalmente es una técnica de análisis temático que permite reconstruir temas fraccionados por más de un encabezamiento de materia, así como la adición de aspectos temáticos omitidos en los encabezamientos de materia, pero no en otros datos de la descripción documental tales como: autores, títulos, títulos de serie, notas, etc., pues lo que se revisa para la elaboración del índice a los encabezamientos de materia son las fichas bibliográficas y no las obras.

En términos generales, para la sistematización de cualquier forma de análisis, entendiendo por análisis la separación de las partes de un todo, deben cumplirse dos requisitos:

a) precisar qué es lo que se busca en el análisis (PEN'71), y una vez hecha esta precisión,

b) determinar las categorías en que pueden clasificarse las partes o elementos del todo que con el análisis se van a identificar.

\section{¿Qué se busca?}

En el caso del análisis temático documental no basta decir que se busca el tema tal y como es tratado en un documento puesto que como ya se ha mencionado antes, una de las causas principales de errores, omisiones e inconsistencias en el análisis temático son las diferentes interpretaciones respecto a lo que es un tema y esto se traduce en falta de precisión en lo que se busca.

Patrick Wilson señala que partiendo de los supuestos de que existe un solo tema en un documento y que ese sólo tema puede ser determinado con base en lo que Coates llama el arte de abstraer en una idea toda la complejidad temática de una obra, se suele confundir el "qué es" un tema, con el "acerca de que" es un tema (WIL'68)

Esta confusión entre el "qué es" y el "acerca de qué" es un tema se puede observar también en algunas de las definiciones que se han dado de tema y en las que indistintamente se indica que tema es, o una "proposición o texto...", o "cualquier concepto o combinación de conceptos", o "la idea básica o el objeto principal de pensamiento o atención...”, o "un cuerpo organizado o sistematizado de ideas...".

A partir de algunas de estas nociones y considerando la diferencia entes "qué es" y "acerca de qué", se pueden pensar que tema documental "es" una proposición "acerca de" uno o varios conceptos o ideas organizadas o sistematizadas que constituyen el objeto principal de atención o pensamiento de un autor.

Existen varios trabajos que se refieren al "acerca de qué" es un tema pero normalmente omiten cualquier consideración sobre el "qué es". Los trabajos más claros que mencionan estos dos elementos temáticos y que explican la diferencia, son el texto programado para instruir en la indización temática de A. G. Brown (BRO'76), y la propuesta de métodos para el análisis temático de documentos de Derek W. Langridge (LAN'89) en la que profundiza, amplía, precisa y categoriza el "qué es" y el "acerca de qué" es un tema documental.

Para A. G. Brown, en el tema de un documento, esa proposición corresponde a lo que él llama, forma intelectual del conocimiento o simplemente forma del conocimiento como prefiere llamarla Langridge.

A los conceptos o ideas organizadas y sistematizadas que constituyen el objeto principal de atención o pensamiento de un autor, Brown los llama conceptos temáticos, Langridge los llama tópicos.

Para Brown y Langridge; las formas del conocimiento y los conceptos temáticos o tópicos que se pueden observar en un tema, son los dos elementos esenciales y característicos que lo conforman y que un análisis debe diferenciar e identificar claramente. Para Brown y Langridge, las formas del conocimiento (la proposición) se refieren a la forma o modo de comprensión o percepción de las cosas (tópicos o conceptos temáticos), es decir, las formas del saber y que, por ejemplo, Aristóteles redujo a tres, el saber teórico, el saber práctico, y el saber poyético o productivo. Como de estas formas de conocimiento o saber que representan el "qué es" un tema derivan las ciencias y disciplinas, generalmente la respuesta al "qué es" suele darse a través 
del nombre de una ciencia o disciplina. Dicho en otra forma, cuando se busca cuál es el tema de un documento, lo que se busca identificar es la forma de conocimiento o disciplina desde la cual se desarrolla o tratan los tópicos o conceptos temáticos.

Los tópicos o conceptos temáticos (conceptos o ideas organizados o sistematizados que constituyen el objeto principal de atención o pensamiento de un autor) se refieren a cualquier cosa que perciba o imagine el ser humano y pueden ser, en consecuencia, infinitos, por ejemplo: el sonido; la lluvia ácida; la belleza; un tratado de libre comercio entre tres naciones; el acondicionamiento técnico para la instalación de un sistema de riego por goteo en zonas semiáridas de un país pobre; la adolescencia; las bibliotecas; la conducta de los delfines en cautiverio, etc. Los tópicos o conceptos temáticos corresponden al "acerca de qué" es un tema, y este "acerca de qué" como pregunta se responde, con el nombre del tópico, independientemente de si ese nombre es, lingüística o conceptualmente, conciso o extenso, simple o complejo.

Para ejemplificar el significado de estos dos elementos esenciales en un tema, considérense dos obras que tratan sobre los problemas de la extracción del petróleo desde yacimientos marinos. Una de estas obras se refiere a los problemas legales de la extracción, y la otra se refiere a los problemas técnicos de la extracción.

En la primera obra, el tema "es" Legislación, y en la segunda "es" Tecnología. Por otra parte en ambas obras el tema es "acerca de" la extracción del petróleo desde yacimientos marinos. Naturalmente, aun cuando los conceptos temáticos o tópicos percibidos o tratados son los mismos en las dos obras, si estas obras se examinan y comparan, son totalmente diferentes. Son tratamientos diferentes porque tratándose de los mismos conceptos temáticos, se perciben, obser- van, cuestionan, afirman o niegan desde formas de conocimiento o experiencia tan diferentes como las del conocimiento legal y el conocimiento tecnológico.

Otro elemento con el que suelen confundirse las formas de conocimiento y los tópicos o conceptos temáticos, es el destinatario, campo de interés o aplicación de un tema cuando ese destinatario no corresponde al campo o grupo de personas que comúnmente están interesados en la forma de conocimiento, o los tópicos que se dan en un tema documental. Es natural que un tema de naturaleza científica interese, o esté destinado a los científicos, que los científicos sean los primeros interesados en consultar el tema, y que sean los científicos las personas mejor preparadas para consultar un documento de tema científico; cuando esto ocurre, y suele ocurrir la mayoría de las veces, es innecesario buscar identificar y representar al destinatario, o el campo de interés o aplicación de un tema, es decir, el “para qué o quién” es un tema cuando ese "para qué o quién" está implícito en la forma de conocimiento.

La confusión entre la forma del conocimiento y el destinatario o aplicación ocurre cuando el destinatario, o la aplicación, previstos o no por el autor, son diferentes a os esperados o comunes. Por ejemplo, en el documento titulado Tipobibliotecografía de Max Díaz y Luz Marina Quiroga quienes presentan una aplicación del programa de tipografía automatizada TEX para la edición de fichas bibliográficas tomando como ejemplo la edición de la Bibliografía Mexicana el análisis temático del documento puede diferenciar tres elementos:

El tema del documento Tipobibliotecografía "es" computación "acerca" de TEX para la edición de fichas bibliográficas "destinado" a aplicaciones bibliotecarias y tipográficas. Decir que el tema "es" de bibliotecología, o tipografía, significa confundir el "para qué o quién"

\begin{tabular}{|c|c|c|}
\hline ¿Qué es? & Computación & (forma de conocimiento) \\
\hline ¿Acerca de qué es? & $\begin{array}{l}\text { TEX para la edición } \\
\text { de fichas bibliográficas }\end{array}$ & $\begin{array}{l}\text { (tópico o conceptos } \\
\text { temáticos) }\end{array}$ \\
\hline ¿Para qué o quién? & $\begin{array}{l}\text { Bibliotecología o } \\
\text { tipografía automatizada }\end{array}$ & (destinatario o aplicación) \\
\hline
\end{tabular}

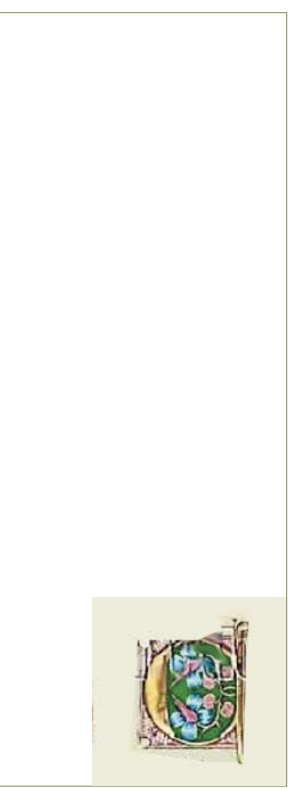

\section{Cuando se busca cuál es el tema de un documento, lo que se busca identificar es la forma de conocimiento o disciplina desde la cual se desarrolla o tratan los
tópicos o conceptos temáticos. la cual se desarrolla o tratan los
tópicos o conceptos temáticos.}


con la naturaleza esencial del tema. Esta suele ser una de las confusiones más comunes y que mayor desconcierto e insatisfacción provoca en los usuarios.

En el caso del ejemplo, el hecho de que el documento pueda estar destinado, o interese más a bibliotecarios y atipógrafos, puesto que percibe y maneja conceptos temáticos o tópicos comunes en la bibliotecología y la tipografía como pueden ser los detalles sobre la forma, naturaleza y posición de los campos o elementos descriptivos en una ficha bibliográfica, su diferenciación tipográfica, y su arreglo y presentación en las cuartillas impresas de una bibliografía, realmente no afecta la naturaleza de la forma de conocimiento desde la que se perciben y tratan los tópicos o conceptos temáticos bibliotecarios y tipográficos. Los conceptos tópicos acerca de la edición de fichas bibliográficas en este documento de Tipobibliotecografía, se perciben y manejan con los métodos, procedimientos y recursos de matemáticas aplicadas esenciales en el conocimiento creativo y productivo de la tecnología computacional.

El "para qué o quién”, no suele afectar la naturaleza esencial de un tema, sino su presentación externa.

En el terreno de la presentación externa de un tema documental, además del elemento destinatario, Brown y Langridge mencionan una amplia gama de elementos que llaman precisamente formas de presentación y que se refieren al "cómo" se presenta un tema.

Entre los elementos o características temáticas agrupadas bajo la denominación de formas de presentación están: los destinatarios o campo de aplicación de un tema; el nivel de tratamiento que el autor desea o puede dar a su discurso o exposición; el idioma o símbolos empleados para comunicar el tema; el tipo de documento y la forma estructural o literaria en que se organizan los datos que se manejan en la exposición. Ejemplo:

Independientemente de casos en que se confunde, o se da prioridad en la representación temática a una o varias formas de presentación sobre las formas de conocimiento, y los tópicos o conceptos temáticos muchas formas de presenta-

Catecismo popular para adultos:

Doctrina cristina.- 16ª . Ed. - México: Asociación Cultural Onir, 1985.

Forma de conocimiento: Religión cristiana católica

Tópicos o conceptos temáticos: Creencias. Credo o fe

Destinatario: Adultos

Nivel: Divulgación

Idioma: Español

Tipo de documento: Libro o monografía impresa

Forma estructural: Catecismo o Preguntas y respuestas

ción se omiten de la representación temática por estar, como en el caso del ejemplo, implícitas o explícitas en elementos de la descripción externa como títulos, subtítulos, editoriales, etc. y solamente en casos excepcionales se precisan en notas (elemento de la descripción externa documental), o entre los elementos de la descripción temática.

Esta práctica ha sido, además de irregular, peligrosa por las confusiones que se han venido mencionando y que afectan la identificación y representación de elementos esenciales en la caracterización de un tema. Una de las críticas que se hacen a los encabezamientos de materia asignados en la Biblioteca del Congreso, se refiere precisamente a esta práctica de omitir elementos o aspectos significativos de un tema porque esos elementos ya están en alguna parte de la descripción externa.

Los problemas de omisión e inconsistencia en el manejo de las formas de presentación como elementos o características temáticas, se han magnificado en los catálogos, bibliografías e índices automatizados cuando éstos son simplemente las mismas fichas bibliográficas de los catálogos en tarjetas, o en cuartillas impresas, trasladadas a un "ambiente" de registros no visibles al ojo humano, solamente "legibles a la máquina" y en donde la recuperación es por "campos" o, en el caso de las representaciones temáticas con simbología verbal, por palabras que se hacen visibles en pantalla, aisladamente, desarticuladas, fuera de su contexto.

En el oscilar de las formas de presentación temática entre los elementos propiamente temáticos y los elementos de des- cripción externa, reconociendo su importancia en el análisis y representación temática, algunos autores prefieren llamar al análisis temático, análisis de contenido por incluir elementos no estrictamente temáticos. En este trabajo y siguiendo el ejemplo de Langridge, aquí se seguirá la tradición bibliotecaria de llamar análisis temático al análisis que se lleva a cabo para identificar elementos esenciales y no esenciales que caracterizan a un tema documental.

En consecuencia, si un tema documental se compone y caracteriza por un conjunto de elementos llamados formas de conocimiento, tópicos o conceptos temáticos, y formas de presentación, es decir elementos que indican "qué es", "acerca de qué es" y "cómo se presenta" un tema, se puede concluir que:

$\left.1^{\circ}\right)$ Tema documental es una forma de conocimiento o saber acerca de un tópico, o combinación organizada de conceptos que atrae la atención especial de un autor dependiendo de la forma de conocimiento o saber del autor, de sus recursos de expresión, y del grupo de personas a quienes desea comunicar sus observaciones sobre un tópico, un tema se presenta en un tipo específico de documento, y dentro de una estructura o género literario determinado y una organización también determinada. En base a esto:

$2^{\circ}$ ) El análisis temático documental busca identificar formas de conocimiento y/o tópicos y/o formas de presentación que caracterizan y de las que se compone un tema documental. 
Considerando como elemento o características temáticas esenciales de un tema documental las formas de conocimiento o saber (formas de pensar) y los tópicos, o conceptos temáticos (contenidos de la forma de pensar), se pueden reconocer dos tipos de sistemas de organización y representación temática documental:

a) Los sistemas de organización y representación temática en los que los tópicos o conceptos temáticos spm subordinados, o deben estar presididos por la forma de conocimiento a la que pertenecen o desde la cual son tratados. Es el caso de las clasificaciones lógicas o sistemáticas generales, enumerativas o facetadas como la Clasificación Decimal de Dewey, la LCC, la Clasificación Colón de Ranganathan, o la Clasificación Bibliográfica de Henry E. Bliss, por citar los más conocidos. En estos sistemas, que no están totalmente relegados al ordenamiento físico de documentos en estantería, pues también se emplean en la Organización de numerosas bibliografías y catálogos impresos (sobrevivencia de los catálogos sistemáticos), las formas de conocimiento a las que estos sistemas se aproximan a través de sus llamadas "clases principales", deben presidir toda representación temática. En estos sistemas ningún tópico puede darse en el vacío, y su significado y alcance depende de las jerarquías y relaciones lógicas establecidas o reconocidas por las ciencias y disciplinas. En estos sistemas, la representación temática debe seguir el orden de: primero el "qué es" y luego el "acerca de qué” es.

b) Y los sistemas de organización y representación temática en los que las formas de conocimiento o saber no importan, se omiten, o relegan a una segunda posición en la organización y representación temática. Es el caso de los encabezamientos de materia, los tesauros, y las clasificaciones facetadas especializadas. Son los sistemas que buscan y proclaman el "acceso directo”, es decir, acceso directo a tópicos o conceptos temáticos. En estos sistemas, primero y ante todo importa identificar y representar el "acerca de qué" es un tema y, después, si es necesaria, la forma de conocimiento.

A partir de los dos tipos de sistemas que claramente revelan por su estructura y símbolos de representación dos claras tendencias en la concepción temática documental, esas estructuras y símbolos de alguna forma requieren dos tipos de análisis temático en los documentos. En las llamadas clasificaciones sistemáticas generales es fundamental buscar y diferenciar tanto forma de conocimiento, como tópico, o conceptos temáticos; en los encabezamientos de materia, tesauros y clasificaciones facetadas especializadas, toda la atención del análisis documental se centra en el tópico, o conceptos temáticos. Entendiendo estas estructuras, es posible mediante un análisis temático independiente, si no mejorar las deficiencias o limitaciones que necesariamente tienen sistemas específicos de clasificación o indización, sí mejorar su comprensión y aplicación.

\section{NOTAS}

1 Considerados prácticamente como sinónimo de tema, los nombres de materia, asunto, argumento, tópico y texto, las definiciones parcialmente citadas corresponden a las definiciones de Juan B. Iguíniz quien define tema, del latín theme, como "proposición o texto que se toma por asunto o materia de un discurso"; la norma ISO 5963-1985 que define materia [subject] como "cualquier concepto o combinación de conceptos que representan el tema [theme] en un documento"; el Webster's new dictionary of synonyms que registra como sinónimo subject, matter, subject-matter, argument, topic, text, theme, motive, motif, y leit motive, indica que éstos "pueden significar la idea básica o el objeto principal de pensamiento o atención en un discurso o composición artística", y finalmente, A. Neelameghan en la Encyclopedia of library and information science (vol. 5, p. 151) define tema [subject] como "un cuerpo organizado y sistematizado de ideas cuya extensión, intenci $\{$ on o coherencia depende del campo de interés, preferencia o competencia intelectual, o especialización de una persona”.

\section{BIBLIOGRAFÍA}

BRO'76 Brown, A. G. Subject analysis and practical classification, en: Introduction to subject indexing: a programmed text, v. i. London. Clive Bingley, 1976.

DUV'81 Duverger, Maurice. Métodos de las ciencias socials. Barcelona. Ariel, 1981, p. 169. 
ISO'88 ISO 5963-1985 (E). Documentation: methods for examining documents, determining their subject, and selecting indexing tems. - en: ISO standards handbook I. Switzerland:

LAN'89 Langridge, Derek W. Subject analysis: principles and procedures. London: Bowker-Saur, 1989.

NEE'71 Neelameghan, A., Classification, theory of., en: Enciclopedia of Library and Information Science. New York: Marcel Dekker, 1971. vol. 5, p. 151.

PEN'71 Penland, Patrick R. Content analysis, en: Enciclopedia of Library and Information Science New York: Marcel Dekker, 1971. vol. 5, p. 632-325.

WIL'68 Wilson, Patrick. Subject and the sense of position, en: Theory of subject analysis: a sourcebook ed, by L. M. Chan, P. A. Richmond, E. Svenonius. Littleton, CO.: Libraries Unlimited, 1985. p. 308-325.

YOU'90 Young, J. B. “Crisis in cataloguing revisted: the year's work in subject analysis, 1990". Library resources \& technical services. Vol. 35(3) 1990: p. 265-282. 